\title{
Prostaglandin E receptor EP4 is a therapeutic target in breast cancer cells with stem-like properties
}

\author{
Namita Kundu $\cdot$ Xinrong Ma $\cdot$ Tyler Kochel $\cdot$ Olga Goloubeva Paul Staats \\ Keyata Thompson - Stuart Martin · Jocelyn Reader • Yukinori Take • \\ Peter Collin · Amy Fulton
}

Received: 12 July 2013/Accepted: 15 November 2013/Published online: 27 November 2013

(C) The Author(s) 2013. This article is published with open access at Springerlink.com

\begin{abstract}
The cyclooxygenase pathway is strongly implicated in breast cancer progression but the role of this pathway in the biology of breast cancer stem/progenitor cells has not been defined. Recent attention has focused on targeting the cyclooxygenase 2 (COX-2) pathway downstream of the COX-2 enzyme by blocking the activities of individual prostaglandin E (EP) receptors. Prostaglandin E receptor 4 (EP4) is widely expressed in primary invasive ductal carcinomas of the breast and antagonizing this receptor with small molecule inhibitors or shRNA directed to EP4 inhibits metastatic potential in both syngeneic and xenograft models. Breast cancer stem/progenitor cells are defined as a subpopulation of cells that drive tumor growth, metastasis, treatment resistance, and relapse. Mammosphere-forming breast cancer cells of human (MDA-MB231, SKBR3) or murine $(66.1,410.4)$ origin of basal-type, Her-2 phenotype and/or with heightened metastatic
\end{abstract}

N. Kundu $\cdot$ P. Staats $\cdot$ A. Fulton

Department of Pathology, University of Maryland, Baltimore, MD, USA

e-mail: nkundu@umaryland.edu

P. Staats

e-mail: pstaats@umm.edu

N. Kundu $\cdot$ X. Ma $\cdot$ T. Kochel $\cdot$ O. Goloubeva $\cdot$ P. Staats .

K. Thompson · S. Martin · J. Reader · A. Fulton ( $\square)$

Greenebaum Cancer Center, University of Maryland,

655 W. Baltimore St., Baltimore, MD 21201, USA

e-mail: afulton@umaryland.edu

X. Ma

e-mail: Xma001@umaryland.edu

T. Kochel

e-mail: tkoch001@umaryland.edu

O. Goloubeva

e-mail: ogoloubeva@som.umaryland.edu capacity upregulate expression of both EP4 and COX-2 and are more tumorigenic compared to the bulk population. In contrast, luminal-type or non-metastatic counterparts (MCF7, 410, 67) do not increase COX-2 and EP4 expression in mammosphere culture. Treatment of mammosphere-forming cells with EP4 inhibitors (RQ-15986, AH23848, Frondoside A) or EP4 gene silencing, but not with a COX inhibitor (Indomethacin) reduces both mammosphere-forming capacity and the expression of phenotypic markers (CD $44^{\text {hi }} / \mathrm{CD} 24^{\text {low }}$, aldehyde dehydrogenase) of breast cancer stem cells. Finally, an orally delivered EP4 antagonist (RQ-08) reduces the tumor-initiating capacity and markedly inhibits both the size of tumors arising from transplantation of mammosphere-forming cells and phenotypic markers of stem cells in vivo. These studies support the continued investigation of EP4 as a potential therapeutic target and provide new insight regarding the

\author{
K. Thompson \\ e-mail: kethompson@som.umaryland.edu \\ S. Martin \\ e-mail: ssmartin@som.umaryland.edu \\ J. Reader \\ e-mail: jread001@umaryland.edu \\ Y. Take \\ AskAt Inc., RaQualia Pharma Inc., Aichi, Japan \\ e-mail: yukinori.take@askat.co.jp \\ P. Collin \\ Coastside Bio Resources, Stonington, ME, USA \\ e-mail: pcollin48@gmail.com \\ A. Fulton \\ Baltimore Veterans Administration, Baltimore, MD, USA
}


role of EP4 in supporting a breast cancer stem cell/tumorinitiating phenotype.

Keywords COX-2 - Prostaglandin E .

Prostaglandin E receptor EP4 - Breast cancer - Stem

cells · Tumor-initiating cells

$\begin{array}{ll}\text { Abbreviations } \\ \text { ALDH } & \text { Aldehydedehydrogenase } \\ \text { COX-2 } & \text { Cyclooxygenase 2 } \\ \text { EP4 } & \text { Prostaglandin E receptor } 4 \\ \text { PGE }_{2} & \text { Prostaglandin } E_{2} \\ \text { MS } & \text { Mammosphere }\end{array}$

\section{Introduction}

Elevated cyclooxygenase 2 (COX-2) expression is common in breast cancer and is associated with a worse prognosis $[1,2]$ but the role of COX-2 pathway members in the behavior of breast cancer stem cells has yet to be defined. The principle COX-2 product in tumors is prostaglandin $\mathrm{E}_{2}\left(\mathrm{PGE}_{2}\right)$ which mediates cellular responses by acting on a family of four $G$ protein-coupled receptors (EP1-EP4). Prostaglandin E receptor 4 (EP4) is expressed in a wide range of epithelial malignancies [3-12] and pharmacologic blockade or genetic silencing of the EP4 receptor inhibits proliferation and migration in vitro and growth and metastasis in vivo [13-17]. These data provide evidence that EP4 and COX-2 are important to the behavior of the general population of tumor cells. Recently, mesenchymal stem cells were shown to create a supportive microenvironment for cancer stem cells by a $\mathrm{PGE}_{2^{-}}$ dependent mechanism [18]. We asked if the COX-2 pathway is supportive of breast cancer stem cell survival by examining the expression and function of COX-2 and EP4 in cells with a stem cell phenotype. We now show that both EP4 and COX-2 are highly induced on candidate tumorinitiating/stem cell populations and that EP4 antagonists reduce cancer stem cell properties in vitro and in vivo supporting the hypothesis that EP4 and/or COX-2 may represent novel targets expressed by the most high risk and resistant subpopulations.

\section{Methods}

Cell lines and mice

Murine mammary tumor lines 66.1 and 410.4 are highly tumorigenic and metastatic in syngeneic Balb/cByJ mice; lines 67 and 410 are poorly tumorigenic and non-metastatic
Fig. 1 a A tissue microarray was prepared containing 44 invasive ductal carcinoma of the breast. EP4 and H\&E by immunohistochemistry. (i) Benign lobule, EP4, 1+; (ii) $\mathrm{H} \& \mathrm{E}$; (iii) invasive ductal carcinoma, EP4, 1+; (iv) H\&E; (v) invasive ductal carcinoma, EP4, 3+; (vi) H\&E. b Line 410.4 tumor cells injected proximal to the mammary fat pad of Balb/cByJ female mice treated with vehicle or RQ-08 (30 mg/kg/day). When tumors measured $18 \mathrm{~mm}$ in diameter, mice were euthanized and surface lung tumor colonies enumerated. Mean $\pm \mathrm{SE}, P=0.04$. c MDA-MB-231-luciferase cells treated with RQ-15986 $(3.0 \mu \mathrm{M} / \mathrm{l})$ or DMSO vehicle and injected i.v. into groups of five Balb/SCID mice and live animal imaging carried out at $5 \mathrm{~min}$ and at the days indicated. Data expressed as percent photons detected relative to day 0 . d Line 66.1 cells transfected with plasmid expressing shEP4 or vector; stable clones were derived and EP4 expression characterized by qPCR. e Cell lines from $\mathbf{d}$ injected i.v. into 5-10 Balb/cByJ female mice and surface lung tumor colonies quantified. Mean $\pm \mathrm{SE}, P<0.01$

in the same hosts. Human breast cancer cell lines MDAMB-231, SKBR3, and MCF7 were obtained from ATCC. The EP4 antagonists AH23848 (Sigma Chem. Co, St. Louis, MO) and RQ-00015986, hereafter abbreviated RQ15986 and RQ-08 (gifts of RaQualia Pharma, Inc., Ref. 19 as CJ-042794), Frondoside A (a gift of Coastside Bio Resources, Ref. 20), indomethacin (Sigma) were added to cell cultures to achieve final concentrations as indicated. Line 410.4 and 66.1 tumor cells were transfected with a plasmid expressing shRNA targeting the murine EP4 gene or control vector. For some studies, the targeting vector was from OpenBiosystems, Huntsville, AL [21]; for other studies an EP4shRNA was obtained from OriGene, Rockville, MD. MDA-MB-231-luc cells were a generous gift of Dr. Stuart Martin, UMB. Balb/cByJ female mice were purchased from the Jackson Laboratory (Bar Harbor, ME); $\mathrm{Balb} / \mathrm{c} / \mathrm{SCID}$ mice were purchased from Charles Rivers Laboratory (Wilmington, MA). Limiting dilution assays were carried out by injecting the indicated number of cells proximal to the inguinal mammary fat pad. Mice were euthanized on an individual basis when tumors measured $18 \mathrm{~mm}$ in largest diameter and lung surface tumor colonies were counted under a dissecting microscope. Tumor volume was calculated by the formula: $\left(a \times b^{2}\right) \times 0.5236$, where $a=$ longest diameter and $b=$ perpendicular diameter. Lung colonization was evaluated by injecting $1-2 \times 10^{5}$ viable tumor cells i.v. into the lateral tail vein of $\mathrm{Balb} / \mathrm{cByJ}$ female mice. All mice were euthanized on day 18-22 post-transplantation or earlier if moribund. Lungs were examined for tumor colonies.

Live animal imaging

Balb/c/SCID mice were injected i.v. with $1 \times 10^{5}$ MDAMB-231-luc cells treated with $3 \mu \mathrm{M}$ RQ-15986 or DMSO. On the days indicated, sedated mice were injected with DLuciferin (Caliper Life Sci., Hopkinton, MA) and wholebody bioluminescence determined by Xenogen system. 
A

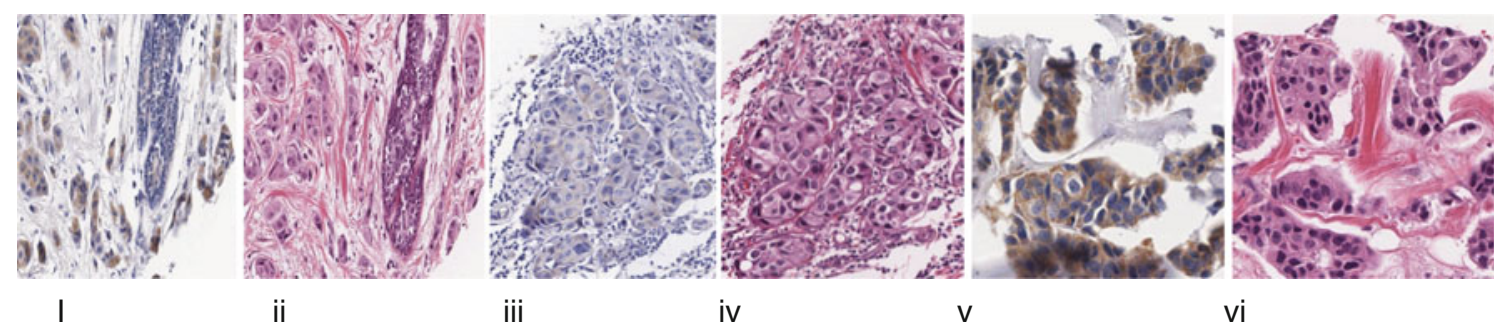

B

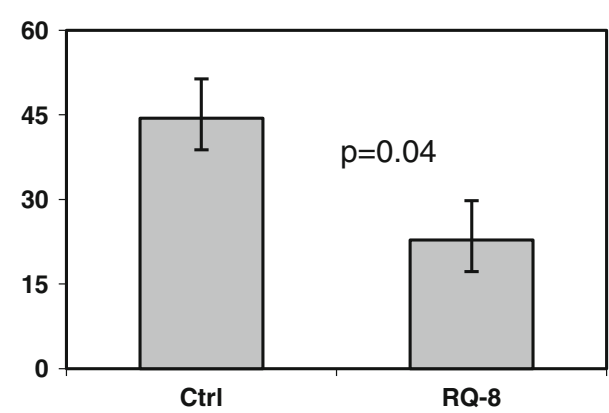

C

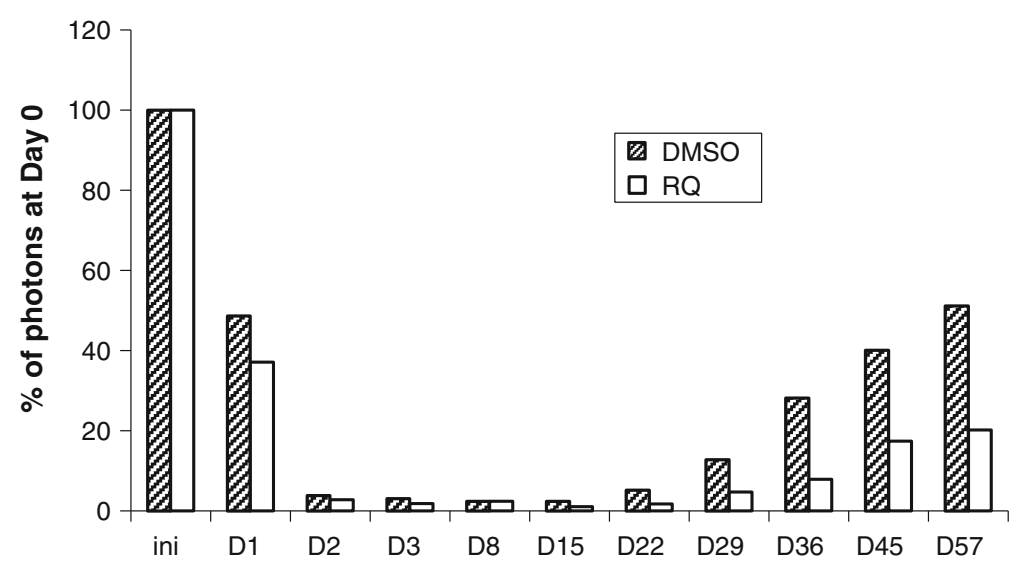

D

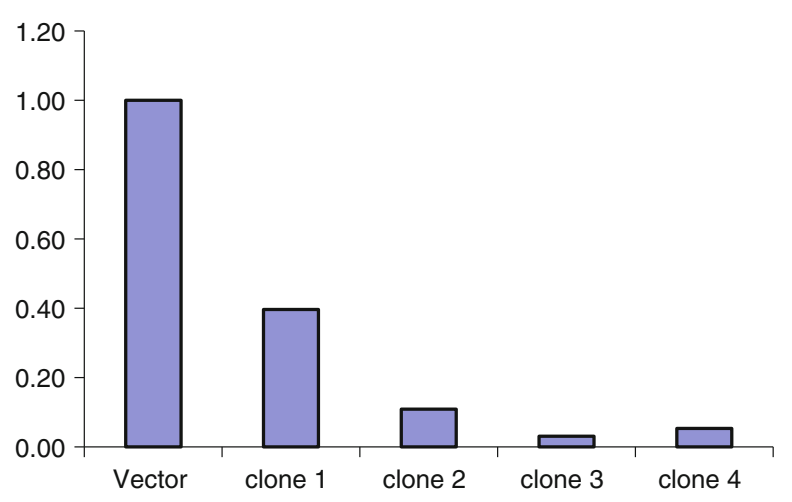

E

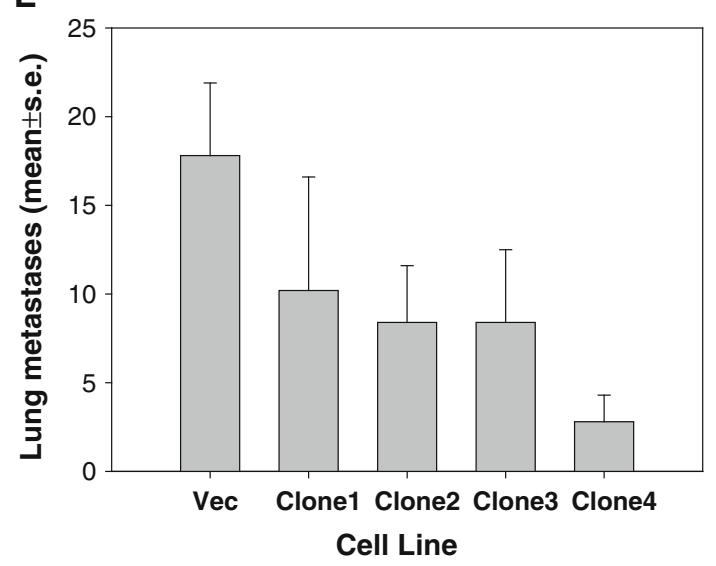


Immunohistochemistry

A tissue microarray was prepared and described previously [22]. Immunohistochemistry was carried out after antigenretrieval using rabbit anti-human polyclonal EP4 antibody (Lifespan Biosciences, Seattle, WA) followed by secondary antibody (EnVision+dual link System, Dako).

\section{qPCR}

Quantitative PCR was conducted using SYBR green (BioRad, Hercules, CA) and 400-700 ng cDNA per reaction. Relative expression levels for COX-2 and EP4 were normalized to GAPDH by the $\Delta \Delta C_{\mathrm{t}}$ method.

\section{Metastasis PCR array}

RNA was extracted from 66.1-vector and 66.1shEP4 cells and analyzed on a mouse tumor metastasis PCR array (Qiagen SABiosciences, Valencia, CA) per manufacturer's protocol.

\section{Western blotting}

Total cellular protein was analyzed for COX-2, EP4, and beta-actin by immunoblotting; Cox-2 (Cayman Chemical, Invitrogen, Ann Arbor, MI), EP4 (Cayman), beta-actin (Sigma AC15), secondary RT for $1 \mathrm{~h}$ [KPL anti-mouse HRP, anti-rabbit HRP (Bio-Rad)] and visualized with ECL SuperSignal West Pico (Pierce Chem. Co., Rockford, IL).

\section{ELISA}

$\mathrm{PGE}_{2}$ levels were determined by $\mathrm{PGE}_{2}$ enzyme immunoassay (Cayman Chemical) and expressed as pg $\mathrm{PGE}_{2} / \mu \mathrm{g}$ total protein.

\section{Mammosphere}

Cells were grown in MammoCult medium (Stem Cell Technologies Inc., Vancouver, CA) supplemented with hydrocortisone, heparin, amphotericin B, and gentamicin (Sigma) and plated in ultra-low attachment plates (Corning Inc., Corning, NY). Three-dimensional spheroidal structures (mammospheres) resulting from the first plating in low-attachment conditions were designated MS-1; subsequent passage into secondary mammosphere cultures at days 7-12 were designated as MS-2 cultures.

Flow cytometry

Cells blocked with $1 \%$ FBS and stained with antibodies recognizing CD44 (FITC-conjugated mouse antihuman
CD44), CD24 (PE-conjugated mouse antihuman CD24) or appropriate isotype control (all from BD Biosciences, San Jose, CA). Aldehyde dehydrogenase was detected by Aldefluor kit (Stem Cell Technologies) used according to manufacturer's protocol and analyzed by FACScan flow cytometry.

\section{Biostatistics}

In vivo data was analyzed by non-parametric KruskalWallis test and pair-wise comparisons were carried out by exact two-sided Wilcoxon test. Tumor incidence data analyzed by generalized linear models approach. Data from in vitro studies analyzed by Student's $t$ test.

\section{Results}

EP4 is widely expressed in primary human breast cancer and targeting EP4 inhibits metastasis

We examined the expression of EP4 in 44 invasive ductal carcinomas of the breast by immunohistochemistry. EP4 expression was very low or absent in normal ducts $(0,1+$, Fig. 1a), malignant epithelium was positive for cytoplasmic EP4 expression. On a scale of $0-3+$ staining intensity, 21/44 (48\%) specimens had 1+ EP4 expression, 13/44 $(29 \%)$ were $2+$ and 10/44 (23\%) were graded as $3+$ in EP4 staining intensity. Nuclear staining was not observed.

EP4 gene silencing or receptor inhibition with small molecule inhibitors block metastasis in a syngeneic murine breast cancer model [13, 20, 21, 23]. In this study, we confirmed, using a second tumor cell line and a different EP4 antagonist (RQ-08), that metastasis is inhibited by EP4 blockade. Line 410.4 tumor cells were implanted into syngeneic Balb/cByJ female mice and oral administration of RQ-08 (30 mg/kg $\times 28$ days) was initiated on day +7 . When tumors achieved an average diameter of $18 \mathrm{~mm}$, mice were euthanized and metastatic disease was assessed. The growth of primary tumors was modestly inhibited by RQ-08 (not shown) but spontaneous metastasis to the lungs was reduced by $49 \%$ (Fig. $1 \mathrm{~b}, P=0.04$ ). Metastatic success of human MDA-MB-231-luc cells was also reduced by an EP4 antagonist (Fig. 1c). We studied cell-autonomous effects of EP4 antagonism on the tumor cell alone, by pretreating tumor cells with RQ-15986 (3.0 $\mu \mathrm{M} / \mathrm{l})$ prior to i.v. injection into Balb/SCID mice. At day 1 after i.v. injection of tumor cells, less luciferase signal was detected when EP4 was antagonized. As the surviving tumor cell populations expanded with time, the difference between the two treatment groups became more pronounced. We also created multiple clones of 66.1 expressing EP4shRNA (Fig. 1d). Metastatic potential was reduced by $43,53,53$, and $84 \%$, 
Table 1 Effect of EP4 reduced expression on metastasis-related genes

\begin{tabular}{|c|c|c|}
\hline Symbol & $\begin{array}{l}\text { Fold } \\
\text { down- } \\
\text { regulation }\end{array}$ & Full name \\
\hline Timp2 & -5.22 & Tissue inhibitor of metalloproteinase 2 \\
\hline Csf1 & -4.29 & Colony stimulating factor 1 (macrophage) \\
\hline Tcf 20 & -4.06 & Transcription factor 20 \\
\hline Ewsr1 & -4.01 & Ewing sarcoma breakpoint region 1 \\
\hline Plaur & -3.89 & Plasminogen activator, urokinase receptor \\
\hline Met & -3.53 & Met proto-oncogene \\
\hline Cxcl12 & -3.47 & Chemokine (C-X-C motif) ligand 12 \\
\hline Chd4 & -3.37 & $\begin{array}{l}\text { Chromodomain helicase DNA binding protein } \\
4\end{array}$ \\
\hline Rorb & -2.92 & RAR-related orphan receptor beta \\
\hline Ctbp1 & -2.88 & C-terminal binding protein 1 \\
\hline CD82 & -2.69 & CD82 antigen \\
\hline CD44 & -2.56 & CD44 antigen \\
\hline Hras1 & -2.54 & Harvey rat sarcoma virus oncogene 1 \\
\hline Col4a2 & -2.34 & Collagen, type IV, alpha 2 \\
\hline Itga7 & -2.16 & Integrin alpha 7 \\
\hline $\mathrm{Rb} 1$ & -2.15 & Retinoblastoma 1 \\
\hline Mtss1 & -2.12 & Metastasis-suppressor 1 \\
\hline Kras & -2.10 & $\begin{array}{l}\text { V-Ki-ras2 Kirsten rat sarcoma viral oncogene } \\
\text { homolog }\end{array}$ \\
\hline Ctnna1 & -2.10 & Catenin (cadherin-associated protein), alpha 1 \\
\hline Brms1 & -2.09 & Breast cancer metastasis-suppressor 1 \\
\hline Smad4 & -2.04 & MAD homolog 4 (Drosophila) \\
\hline Etv4 & -1.99 & $\begin{array}{l}\text { Ets variant gene 4(E1A enhancer binding } \\
\text { protein, E1AF) }\end{array}$ \\
\hline Fat1 & -1.89 & FAT tumor suppressor homolog 1 (Drosophila) \\
\hline Rpsa & -1.86 & Ribosomal protein SA \\
\hline Pten & -1.82 & Phosphatase and tensin homolog \\
\hline Mta1 & -1.74 & Metastasis-associated 1 \\
\hline Htatip2 & -1.63 & $\begin{array}{l}\text { HIV-1 tat interactive protein } 2 \text {, homolog } \\
\text { (human) }\end{array}$ \\
\hline $\operatorname{Trp} 53$ & -1.57 & Transformation-related protein 53 \\
\hline
\end{tabular}

RNA isolated from 66.1vector or 66.1 shEP4 cells and analyzed on a mouse Metastasis array. Genes downregulated $>1.5$-fold in 66.1 shEP4 cells relative to 66.1-vector cells are listed

respectively, in comparison to mice injected with vector control cells (Fig. 1e). Thus, EP4 is widely expressed in breast cancer and either genetic or pharmacologic compromise of EP4 activity reduces metastatic potential.

Metastasis and stem cell-associated genes are downregulated in shEP4 cells

We employed a qPCR array of known metastasis-associated genes to compare gene expression patterns of 66.1vector versus $66.1 \mathrm{shEP} 4$ cells. Table 1 shows genes that were downregulated by at least 1.5 -fold in $66.1 \mathrm{shEP} 4$ cells compared to 66.1-vector cells that included Csf1, c-met, CXCL12, and CD44. Few genes were upregulated in the context of EP4 silencing, but included the metastasis-suppressor Nme4 (data not shown). The downregulation of Csf1, Timp2, and CD44 in 66.1shEP4 cells was confirmed by qPCR (Fig. 2a). While each of these gene expression changes may be important to the mechanism of metastasis inhibition, we focused our further studies on the reduction in CD44, a phenotypic marker of candidate breast cancer stem/tumor-initiating cells [24, 25].

Tumor-initiating cells are identified in a syngeneic model of metastatic breast cancer

A true cancer stem cell should have heightened tumorinitiating capacity compared to the general population but a tumor-initiating cell has not been demonstrated in the murine cell lines under study. We cultured 410.4 cells in low-attachment conditions to demonstrate that mammospheres will form. These mammospheres can be dissociated and replated to form secondary and tertiary mammospheres (dns). Limiting dilution assays compared the tumorigenic properties of the general population of 410.4 cells versus mammosphere-forming (MS-1) 410.4 cells. Three of 10 mice injected with 100 of 410.4 bulk cells developed palpable tumors (dashed lines), whereas 8/9 mice injected with MS-1 cells (solid lines) developed progressively growing tumors (Fig. 3c). In an expanded limiting dilution assay using additional cell injection doses (Table 2), five of eight mice $(62.5 \%)$ injected with 100 MS-1 developed tumors and an average of 17 metastases per mouse; only $2 / 8$ mice $(25 \%)$ injected with 100 cells of the bulk population developed tumors and an average of 0.5 metastases per animal, indicating heightened tumorigenic capacity of mammosphere-forming cells. At all cell doses, MS-1 cells were more tumorigenic than the same number of bulk population cells; the stem cell frequency was calculated as 1/145 MS-1 cells versus $1 / 526$ for bulk cells $(P<0.00425)$. The average number of metastases was also higher in mice injected with MS-1 cells compared to an equal number of bulk population cells.

EP4 and COX-2 are upregulated in mammospheres of aggressive breast cancer cells

Several laboratories have described the stem cell properties of human breast cancer cell lines [26-28] and we confirmed that MDA-MB-231 cells are highly enriched for a stem cell phenotype whereas this population is rare in MCF7 cells, which have a luminal gene signature. Consistent with the literature, more than $95 \%$ of MDA-MB231 cells are $\mathrm{CD} 44^{\mathrm{hi}} / \mathrm{CD} 24^{\text {low }}$ (Fig. $3 \mathrm{a}$ ). In comparison, 
Fig. 2 a mRNA isolated from 66.1-vector and 66.1 shEP4 cells and analyzed for expression of metastasis-related genes by metastasis pcr array. b 410.4 cells grown in standard culture or as mammospheres and on day 10 cells were harvested and 100 MS-1 or standard bulk culture cells injected into the mammary fat pad of Balb/cByJ female mice. Tumor growth monitored by caliper and expressed as tumor volume. Solid line $=100$ MS-1 cells; dashed line $=100$ bulk population cells
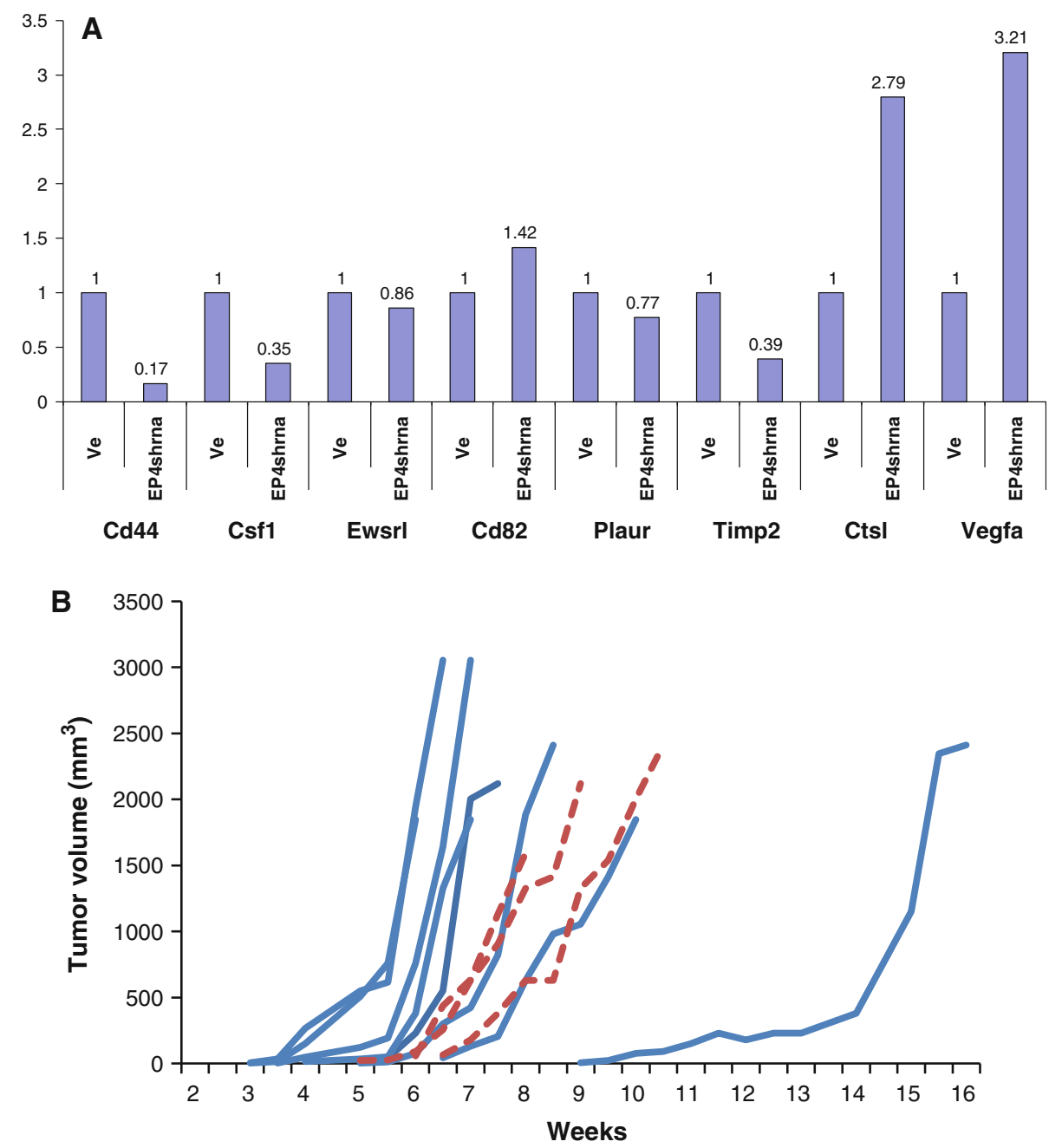

$<1.0 \%$ of MCF7 cells are CD44 ${ }^{\text {hi }}$ (Fig. 3b), however, these latter cells are highly positive for CD24 expression. The fraction of aldehydedehydrogenase $(\mathrm{ALDH}+)$ positive cells was highly variable from experiment to experiment; in one experiment, $5.4 \%$ of MDA-MB-231 cells were $\mathrm{ALDH}+$ (Fig. 3c); $0.23 \%$ of MCF7 cells were ALDH+. Twenty-six percent of 410.4 cells are ALDH+; $57.4 \%$ of 66.1 cells are positive for this marker (data not shown).

We compared EP4 and COX-2 expression levels in mammosphere-forming and bulk populations derived from human or murine breast cancer cell lines. MDA-MB-231 were employed as a model of breast cancer stem cells, MCF7 as a model of non-stem cells and SKBR3 represent the HER-2 phenotype. Metastatic murine cell lines 410.4 and 66.1 and non-metastatic cell lines 410 and 67 were employed for comparison. In both MDA-MB-231 and SKBR3 cells, EP4 mRNA levels were markedly increased in MS-1 versus bulk population cells (Fig. 4a) but EP4 mRNA was not elevated in MS-1-forming MCF7 cells. These patterns have been observed in three independent experiments. Like MDA-MB-231 and SKBR3 cells, murine 66.1 and 410.4 MS-1 cells expressed increased EP4 mRNA versus the bulk population (Fig. 4b), but EP4 was not increased in the comparatively benign 410 and 67 cells in either MS-1 or MS-2 cultures (Fig. 4c). Like EP4, COX2 was increased in MS-1 versus bulk populations of MDAMB-231 and SKBR3 cells (Fig. 4d) but slightly elevated in MCF7 MS-1 cultures. In murine cells, COX-2 was induced in MS-1 cultures of 66.1 and 410.4 (Fig. 4e), but not in 410 or $67 \mathrm{MS}-1$ cells (Fig. 4f). Consistent with the mRNA data, increased COX-2 protein was detected in MS-1 cells of MDA-MB-231 and SKBR3 grown in either DME or mammocult media; only a slight increase in $\mathrm{COX}-2$ protein was detected in MCF-7 cells (Fig. 4g). Increased COX-2 in MS- 1 cells resulted in more $\mathrm{PGE}_{2}$ detected in conditioned media of MDA-MB-231 and SKBR3, but not MCF7derived MS-1 cells (Fig. 4h). Thus, in both human and mouse, EP4 and COX-2 are elevated in mammospheres produced by highly tumorigenic, basal-type cell lines. Luminal-type (MCF7) or less tumorigenic, non-metastatic $(410,67)$ cells do not elevate EP4 or COX-2 expression in MS-1 tumorspheres. 
Fig. 3 a, b MDA-MB-231 and MCF7 cells grown in standard culture conditions and analyzed for CD44 and CD24 expression by flow cytometry. Isotype control (left panel), double stain for CD44 and CD24 (right panel). c Aldefluor assay of MDA-MB-231 (left panel) and MCF7 (right panel) cells

\section{A}

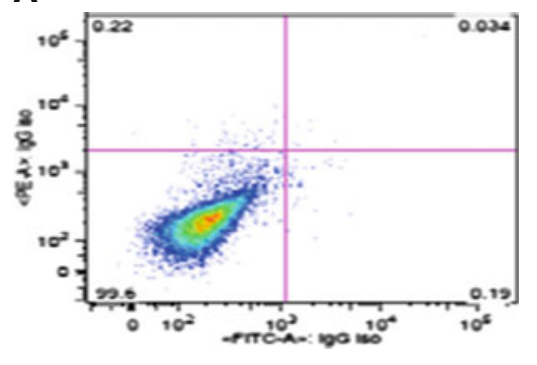

MDA-MB-231, isotype

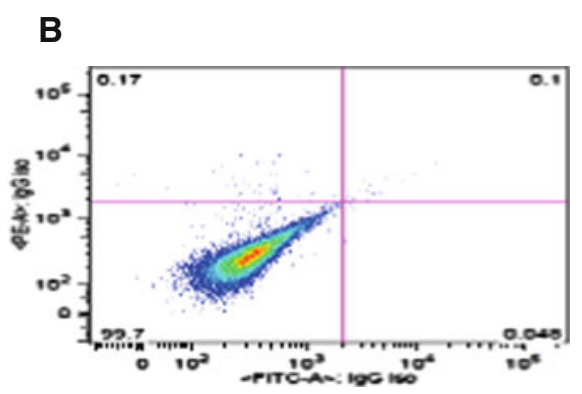

MCF7, isotype

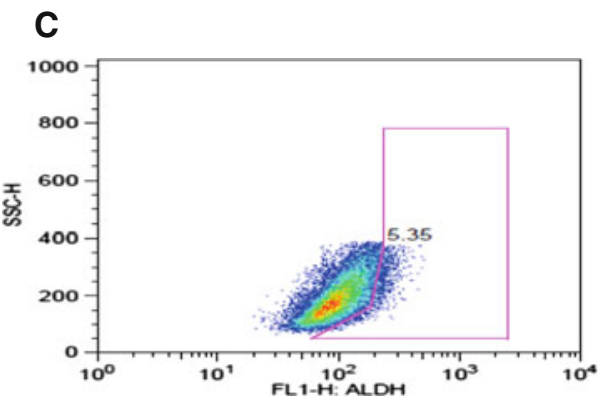

MDA-MB-231, ALDH

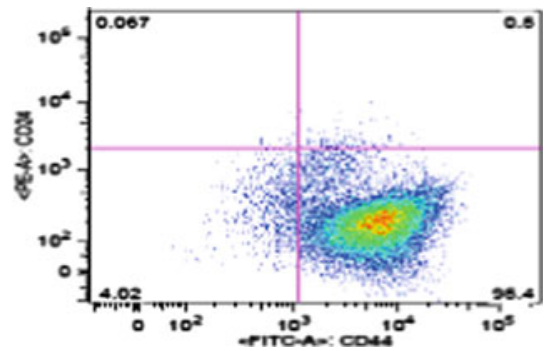

MDA-MB-231, CD44/CD24

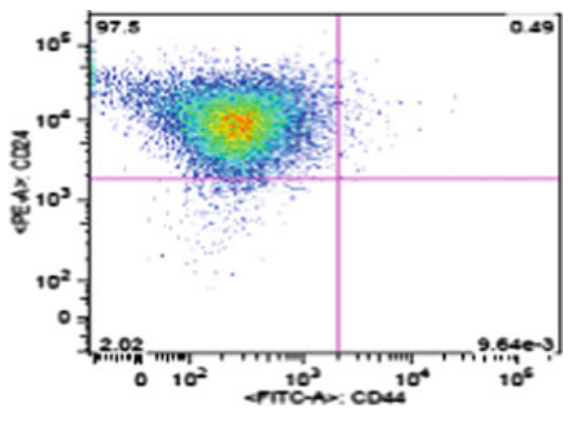

MCF7, CD44/CD24

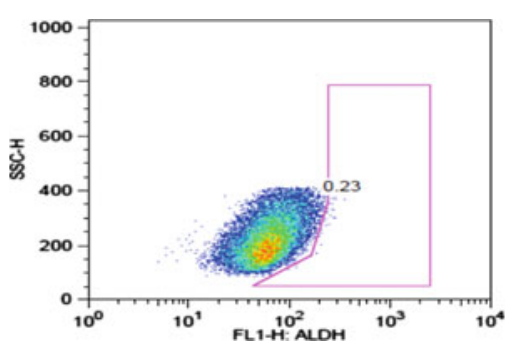

MCF7, ALDH
EP4 antagonists or EP4 gene silencing inhibits properties of breast cancer stem cells in vitro

We compared the growth properties of mammospheres derived from 410.4-vector cells to 410.4shEP4 cells (Fig. 5a). MS-1 mammospheres were disrupted and 5,000 cells from each MS-1 culture was replated and allowed to form secondary (MS-2) mammospheres. The average number of MS-2 spheres formed by 410.4-vector cells $(5.6 \pm 0.9)$ was reduced by $57 \%$ in $410.4 \mathrm{shEP} 4$ mammospheres (2.4 \pm 0.4 spheres). More marked effects of EP4 gene silencing were detected when the size of mammospheres, as indicated by the number of cells/sphere was considered. Mammospheres formed by 410.4-vector, $410.4 \mathrm{shEP} 4,410$ or 67 cells were collected from each well and the total number of sphere-forming cells was determined (Fig. 5a). Five thousand MS-1 410.4-vector cells expanded into secondary tumorspheres to produce a total of
$280,666 \pm 6,992$ cells; a 56-fold expansion in cell number from day 0 . In contrast $410.4 \mathrm{shEP} 4$ cells expanded by 21-fold. Thus, when mammosphere-induced upregulation of EP4 was prevented by shEP4, mammosphere-forming ability (number and size) was compromised. Although line 410 and 67 cells were able to form small secondary mammospheres, the total average cell number was expanded by 12 -fold and 3 -fold, respectively. The reduced sphere-forming ability of 410 and 67 cells is consistent with the failure to upregulate EP4 or COX-2 in mammospheres of these cells.

We determined the effect of EP4 antagonists or the COX-1/COX-2 inhibitor indomethacin on sphere-forming ability of MDA-MB-231 cells. In the presence of indomethacin $(1.0-50 \mu \mathrm{M} / \mathrm{l})$, neither the numbers of mammospheres nor the size of spheres was affected by COX inhibition (Fig. 5b); cell number was $90-115 \%$ of that observed in vehicle-treated TS-1 cells. In contrast, three 
Table 2 Tumor-initiating capacity of mammosphere-forming cells

\begin{tabular}{llll}
\hline Cell type & $\begin{array}{l}\text { Tumor } \\
\text { incidence }\end{array}$ & $\begin{array}{l}\text { \% Tumor } \\
\text { incidence }\end{array}$ & $\begin{array}{l}\text { Lung } \\
\text { mets }\end{array}$ \\
\hline 410.4 MS-1, 10 cells & $1 / 8$ & 12.5 & 7 \\
410.4 Bulk, 10 cells & $0 / 8$ & 0 & 0 \\
410.4 MS-1, 50 cells & $3 / 8$ & 37.5 & 0.3 \\
410.4 Bulk, 50 cells & $1 / 8$ & 12.5 & 0 \\
410.4 MS-1, 100 cells & $5 / 8$ & 62.5 & 17 \\
410.4 Bulk, 100 cells & $2 / 8$ & 25 & 0.5 \\
410.4 MS-1, 500 cells & $7 / 8$ & 87.5 & 3.9 \\
410.4 Bulk, 500 cells & $5 / 9$ & 55.6 & 3.6 \\
410.4 Bulk, 5 $\times 10^{5}$ & $5 / 5$ & 100 & 28.2 \\
\hline
\end{tabular}

MS-1 are mammosphere-derived cells or bulk cells growing under attached culture conditions and injected at the indicated cell doses into the mammary fat pad of syngeneic Balb/cByJ female mice. Tumors palpated twice weekly and degree of metastatic disease determined at necropsy when tumors measured $18 \mathrm{~mm}$ in average diameter

EP4 antagonists, AH23848, Frondoside A and RQ-15986 were all able to inhibit the size of mammospheres (Fig. 5c-e). In the presence of AH23848, mammospheres were actually increased in number, but individual spheres were much smaller as indicated by the dramatic reduction in cells/ sphere. AH23848 at 10, 5, 0.5 , or $0.05 \mu \mathrm{M} / 1$ reduced sphere cellularity by $78,69,55$, and $2 \%$, respectively (Fig. 5 c). In contrast, AH23848 did not affect the number of attached cells at any concentration examined (dns). While the number of total spheres was not affected by Frondoside A except at the highest concentration tested $(1.0 \mu \mathrm{M} / \mathrm{l})$, the sphere size was inhibited by $100,92,28$, and $1 \%$ in a dosedependent manner (Fig. 5d). RQ-15986 (30, 10, 3, and $0.3 \mu \mathrm{M} / \mathrm{l})$ inhibited sphere-forming cells by $20,30,8$, or $7 \%$ (Fig. 5e).

Taken together, these data indicate that tumor cells with heightened stem cell properties are more sensitive than the general population to EP4 antagonism.

EP4 antagonists inhibit the stem cell phenotype in vitro

To determine if EP4 inhibition would downregulate phenotypic markers of breast cancer stem cells, MDA-MB-231 cells were placed in mammosphere culture and on day 8 , RQ-15986 (10 $\mu \mathrm{M} / \mathrm{l})$ was added. On day 10 , cultures were analyzed for ALDH expression. In a typical experiment, RQ-15986 treatment reduced the proportion of $\mathrm{ALDH}^{+}$ MDA-MB-231 cells from $30.4 \%$ to $16.7 \%$ (Fig. 6a). RQ15986, Frondoside A or AH23848 also modestly reduced the percentage of CD44-positive MS-1 cells. In the presence of PBS, $64 \%$ of MDA-MB-231 (MS-1) cells were CD44-positive; Frondoside A $(0.25 \mu \mathrm{M} / \mathrm{l})$ reduced the fraction of CD44-positive cells to $54.3 \%$. In the presence of DMSO, $53.5 \%$ of cells were CD44-positive; RQ-15986 $(10 \mu \mathrm{M} / 1)$ or $\mathrm{AH} 23848(5 \mu \mathrm{M} / \mathrm{l})$ reduced the CD44-positive fraction to 45.9 or $46.9 \%$ of cells, respectively. CD44 expression was not changed in attached cells in the presence of EP4 antagonists (dns). CD24 is poorly expressed in MS- 1 cells and the degree of expression was not changed by treatment with any EP4 antagonist (dns).

EP4 antagonism inhibits tumor-initiating capacity in vivo

By limiting dilution assays, we assessed the ability of an EP4 antagonist to inhibit tumor-initiating cells in vivo. $\mathrm{Balb} / \mathrm{cByJ}$ mice were transplanted with 500 or 50 of 410.4 MS- 1 cells and, beginning on day +7 , were treated with vehicle or RQ-08 for 28 days and tumor incidence and size were assayed. In two independent experiments, EP4 antagonism was able to significantly inhibit the tumorforming capacity of small numbers of tumor cells as indicated by tumor incidence (Fig. 6b). When 500 cells were injected, tumor incidence was reduced from $90 \%$ to $60 \%$ by RQ-08 $(P<0.02)$; RQ-08 inhibited tumor-initiating capacity from $60 \%$ tumor-positive mice to $20 \%$ tumorpositive when 50 cells were injected $(P<0.058)$. The calculated stem cell frequency was reduced from $1 / 126$ cells to $1 / 460$ cells by RQ-08 $(P<0.018)$. While the average size of tumors is sometimes variable reflecting the different latency in individual mice, it is obvious that RQ08 has a profound effect on the ability of palpable lesions to expand (Fig. 6c). In animals that developed palpable tumors in spite of RQ-08, the percentage of tumor cells that were $\mathrm{CD} 44^{\mathrm{hi}} / \mathrm{CD} 24^{\mathrm{low}}$ was reduced from $84.2 \% \pm 1.3$ (vehicle) to $74.1+1.3 \%(P<0.006)$.

\section{Discussion}

EP4 expressed on the malignant cell contributes to metastatic behavior in a cell-autonomous manner as demonstrated by reduced metastatic potential of tumor cells in which EP4 expression has been silenced or antagonized by small molecule inhibitors. Tumor-derived $\mathrm{PGE}_{2}$ also acts to suppress the anti-metastatic activities of NK cells by acting on EP4 expressed on the NK cell [23, 29, 30]. Other EP4-positive immune effector cells are also inhibited by $\mathrm{PGE}_{2}$ [31-33]. Thus, the function of multiple EP4 receptorpositive cells are affected directly by EP4 antagonism.

EP4 promotes multiple cancers [4-17] but little is known about the role of the COX-2 pathway in cancer stem cells. Mesenchymal stem cells are stimulated by IL-1 to produce $\mathrm{PGE}_{2}$ that creates a supportive microenvironment for cancer stem cells [18]. Stromal fibroblasts support the induction of MCF7-derived stem cells; in that model, $\mathrm{PGE}_{2}$ 

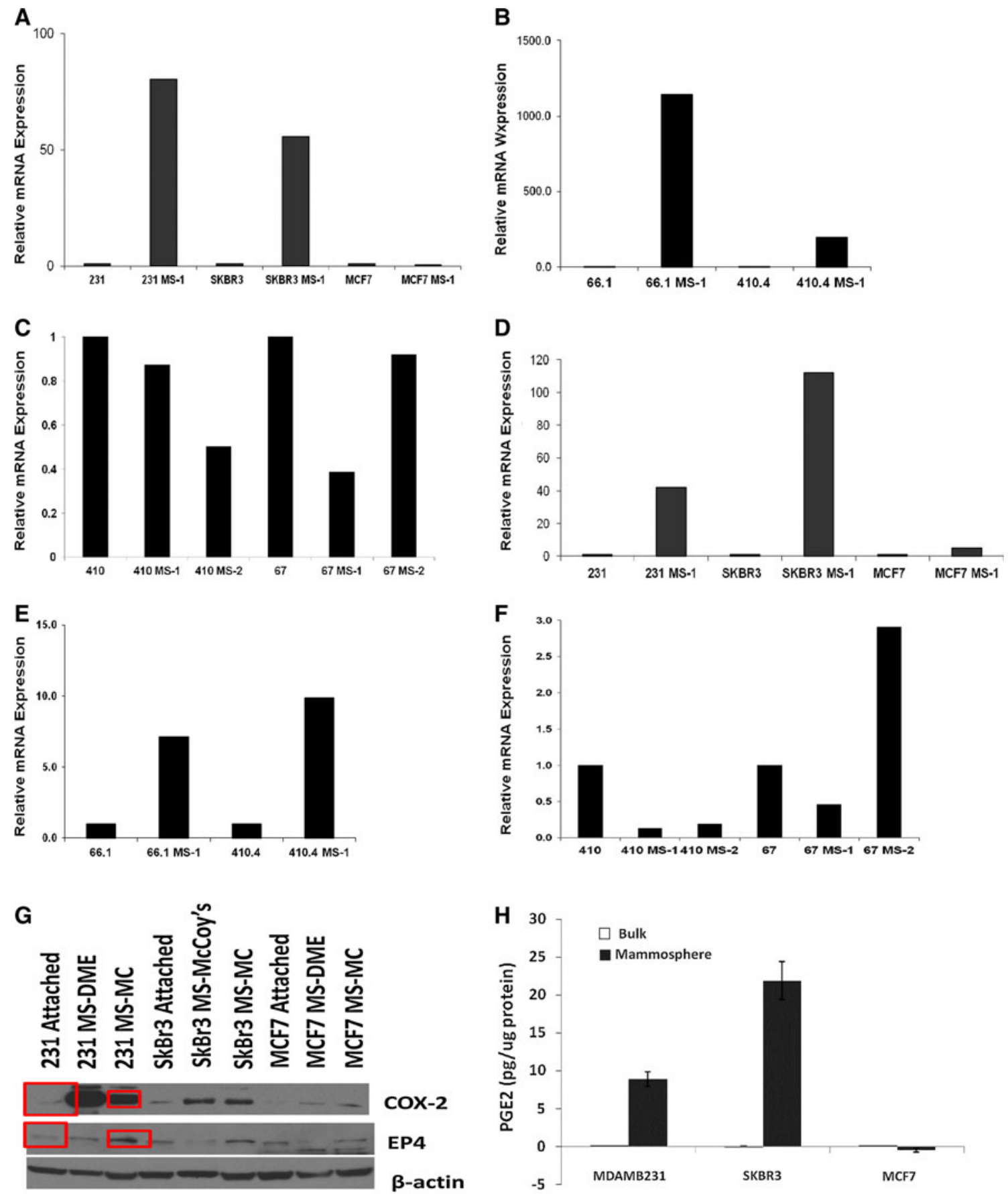

Fig. 4 a MDA-MB-231, SKBR3, or MCF7 cells or b 66.1 or 410.4 or c 410 or 67 cells grown as MS-1, MS-2, or bulk cultures. mRNA harvested and analyzed for human or murine EP4 by qPCR. $\mathbf{d}-\mathbf{f}$ The same cells analyzed for COX-2 mRNA expression. $\mathbf{g}$ Cell lysates of MDA-MB-231, SKBR3, or MCF7 cells grown as standard culture or in mammosphere (MS) assay and maintained in either DME media or

is not sufficient to directly act on the progenitor stem cells [34]; rather an indirect mechanism is proposed in which $\mathrm{PGE}_{2}$ acts indirectly through the cancer-associated fibroblast to induce a stem cell phenotype. The relevant EP receptor was not identified in that study.

mammocult (MC) media and immunoblotted for COX-2, EP4, or $\beta$-actin. h MDA-MB-231, SKBR3, or MCF7 cells grown as attached (open bar) or mammosphere (closed bar) in DME; conditioned media analyzed for $\mathrm{PGE}_{2}$ by ELISA and expressed as Mean $\pm \mathrm{SE}, \mathrm{pg} / \mu \mathrm{g}$ protein

Because mammary tumor cells have elevated endogenous COX-2 activity, we investigated the role of $\mathrm{PGE}_{2}$ and EP4 in an autocrine mechanism that would support breast cancer stem cells. We now show that a sub-population enriched in tumor-initiating cells upregulates both EP4 and 

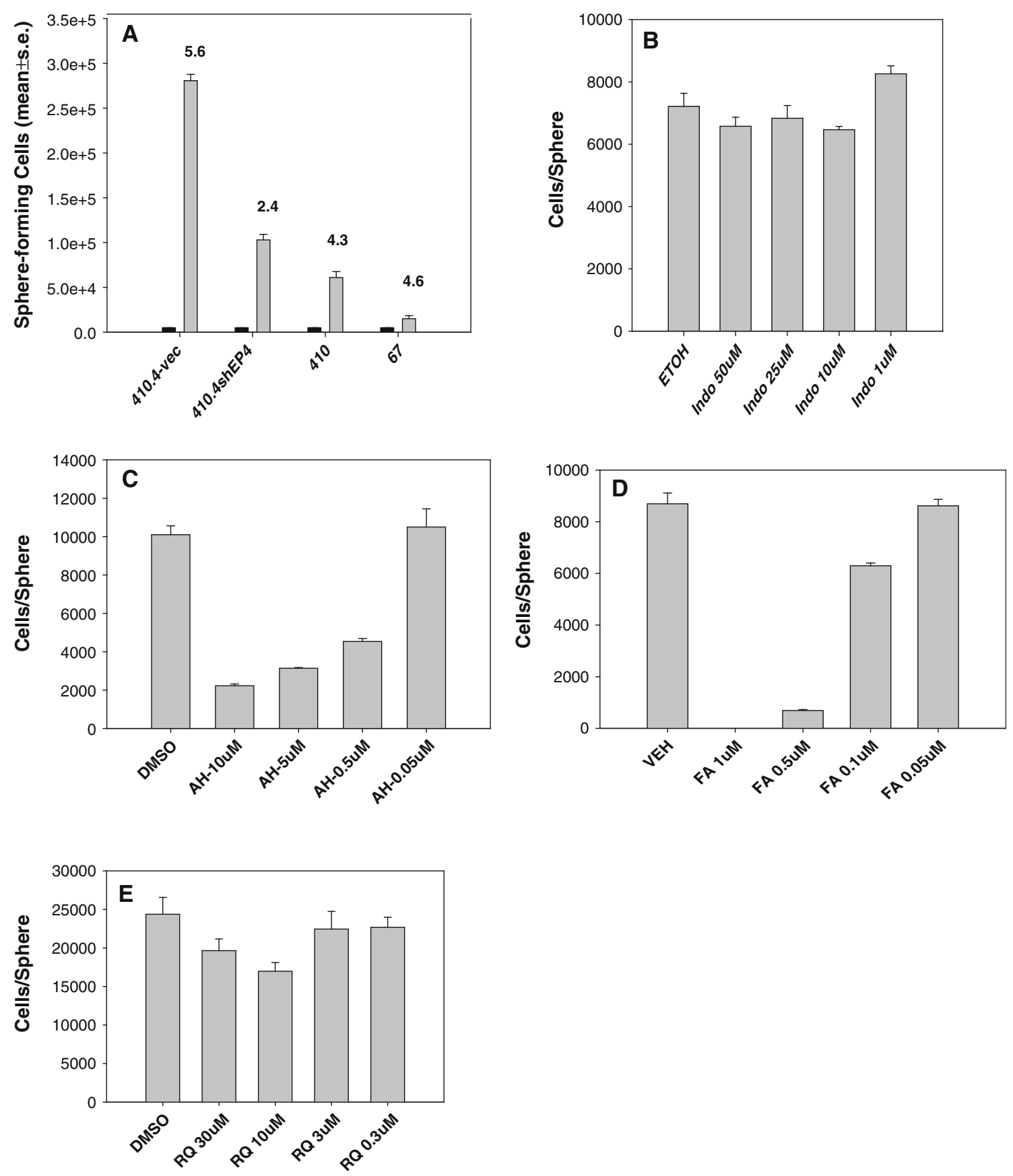

Fig. 5 a $5 \times 10^{3}$ MS- 1 cells of 410.4 -vec, 410.4 shEP4, 410 or 67 cells (black bars) were re-plated in secondary (MS-2) cultures and on day 10 , sphere number and cellularity were determined for MS-2 cultures. Mean \pm SE. b-e MDA-MB-231 cells placed in

COX-2. Upregulation of these genes was only observed in metastatic and/or basal-type human (MDA-MB-231, SKBR3) and murine $(410.4,66.1)$ cell lines but not in nonmetastatic or luminal-type cells suggesting that tumor-initiating cells may contribute to the aggressive phenotype and may be relatively more sensitive than the non-stem cell population to direct inhibition by either EP4 antagonists or COX inhibitors.

mammosphere culture and on day 2, treated with Indomethacin, AH23848, Frondoside A or RQ-15986 at the indicated concentrations. On day 10 , sphere number and cellularity were determined

The upregulation of EP4 expression in mammospheres is functionally linked with increased tumor-initiating cell capacity in vivo of mammosphere-forming cells. MS-1 cells were more tumorigenic than the bulk population with an increase in stem cell frequency. Importantly, tumorigenic potential of MS-1 cells was significantly reduced by an EP4 antagonist in vivo corresponding to a loss of CD $44^{\text {hi }} / \mathrm{CD} 24^{\text {low }}$ cells. In the relatively few RQ-treated 
Fig. 6 a MDA-MB-231 cells in mammosphere culture were treated with vehicle (left panel) or RQ-15986 (10 $\mu \mathrm{M} / 1$, right panel) on day 8 and 2 days later, Aldefluor positive cells were determined. b Five hundred or 50 of 410.4 MS-1 cells were injected into groups of $10 \mathrm{Balb} / \mathrm{cByJ}$ female mice. On day +7 , RQ-08 or vehicle administered by gavage $(30 \mathrm{mg} /$ $\mathrm{kg}$ /day) and tumor incidence on day +46 is plotted. c Tumor volume for the same mice as in b that developed palpable lesions. Mean $\pm \mathrm{SE}$
A
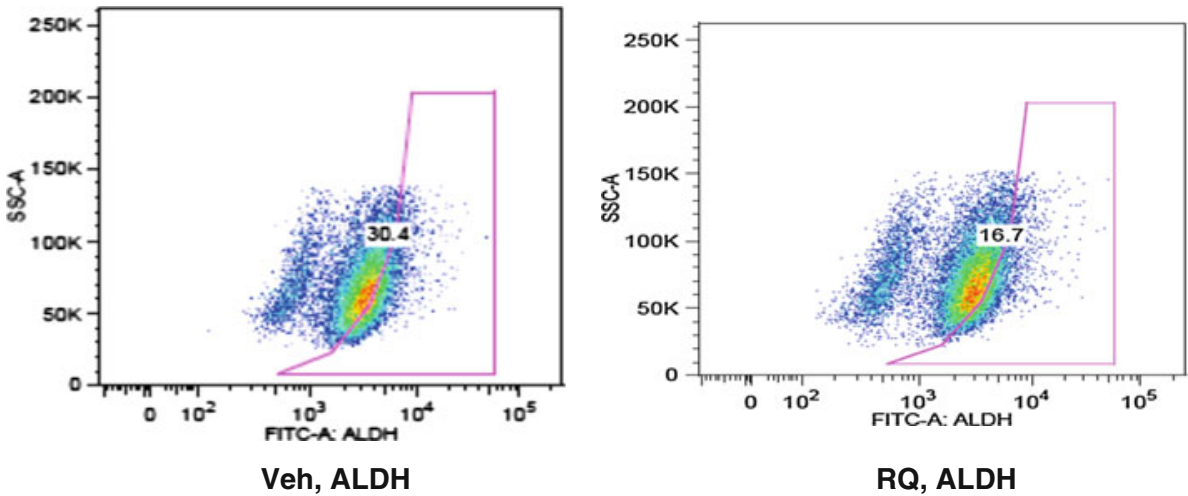

RQ, ALDH
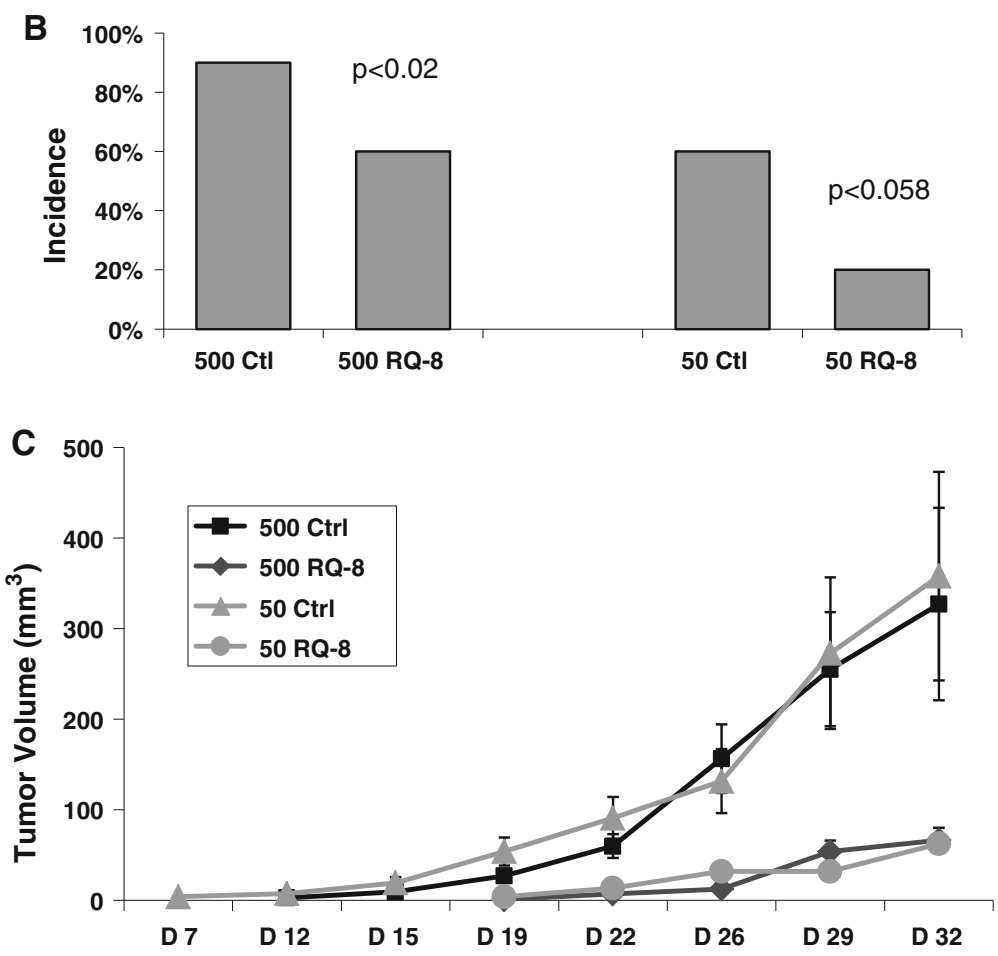

mice that developed palpable lesions, the tumors never expanded. This is in contrast to the modest inhibition of tumor size when bulk tumor cells are injected and is consistent with the increased expression of EP4 in MS-1 cells. Consistent with the studies in vivo, in comparison to 410.4-vector cells, 410.4shEP4 cells were less able to expand to form large mammospheres in culture. Interestingly, growth properties in vitro in conventional attached conditions are not different for EP4 high versus low cells supporting the hypothesis that EP4 has important functions in maintaining the stem/progenitor phenotype. 410.4-vector cells expanded by 56-fold during 10 days of culture under low-attachment conditions; the same number of 410.4shEP4 cells were only able to expand by 21 -fold. Poorly tumorigenic 410 and 67 cell lines produced only small mammospheres. Likewise, the addition of three different EP4 antagonists to mammosphere-forming cultures were each able to inhibit mammosphere growth. Interestingly, the COX-1/COX-2 inhibitor indomethacin did not affect sphere size, indicating that it is not $\mathrm{PGE}_{2}$ synthesis per se, but more likely to be $\mathrm{PGE}_{2}$-mediated cell signaling that supports sphere formation. The fraction of $\mathrm{CD} 44^{\text {hi }}$ or $\mathrm{ALDH}^{+}$cells, both phenotypic markers of tumor-initiating cells, were reduced by each of three EP4 antagonists.

We did not observe appreciable upregulation of COX-2 in MCF7 tumorspheres consistent with the luminal properties of this cell line. Singh et al. detected a rare COX-2 highly positive cell in MS-1 cultures of MCF7; in these cells, the stem cell factor Oct4 was often co-expressed [35]. Those authors propose that the COX $-2^{\text {hi }}$, Oct4+ is a breast cancer stem cell, consistent with the current report. 
Taken together, these studies support the hypothesis that EP4-mediated activation can, in an autocrine or paracrine manner drive stem cell survival. Our studies show, for the first time, that a clinically relevant EP4 antagonist can inhibit breast cancer stem cells. Blocking EP4 inhibits breast cancer metastasis and tumorigenic capacity in vivo and stem cell properties including mammosphere-forming ability and phenotypic markers of breast cancer stem cells in vitro. EP4 antagonism may prove to be safer and more effective than global COX-2 inhibition. The demonstrated efficacy of EP4 antagonists in preclinical models of breast and other malignancies supports the continued evaluation of EP4 as a new therapeutic target in advanced malignancy.

Acknowledgments We thank the Biostatistics, Genomics, Flow cytometry, and Tissue Biorepository Shared Services of the University of Maryland Greenebaum Cancer Center. We thank RaQualia Pharma Inc and Coastside Bio Resources for the gift of EP4 antagonists. These studies were supported by the United States Department of Health and Human Services and the United States Veterans Administration.

Competing interests The authors declare no competing interests.

Ethical statement All studies comply with current laws of the U.S.A. Mice were housed, cared for, and used in strict accordance with the U.S. Department of Agriculture regulations and the NIH Health Guide for the Care and Use of Laboratory Animals. All animal protocols were reviewed and approved by the University of Maryland Institutional Animal Care and Use Committee. The University of Maryland School of Medicine Animal Facility is fully accredited by the American Association for the Accreditation of Laboratory Animal Care.

Open Access This article is distributed under the terms of the Creative Commons Attribution Noncommercial License which permits any noncommercial use, distribution, and reproduction in any medium, provided the original author(s) and the source are credited.

\section{References}

1. Ristimaki A, Sivula A, Lundin J, Lundin M, Salminen T, Haglund $C$ et al (2002) Prognostic significance of elevated cyclooxygenase-2 expression in breast cancer. Cancer Res 62:632-635

2. Kerikowske K, Molinaro AM, Gauthier ML, Berman HK, Waldman F, Bennington J, Sanchez H et al (2010) Biomarker expression and risk of subsequent tumors after initial ductal carcinoma in situ diagnosis. J Natl Cancer Inst 102:627-637

3. Reader JC, Holt D, Fulton AM (2011) Prostaglandin E2 EP receptors as therapeutic targets in breast cancer. Cancer Metastasis Rev 30:449-463

4. Mutoh M, Watanabe K, Kitamura T, Shoji Y, Takahashi M, Kawamori $\mathrm{T}$ et al (2002) Involvement of prostaglandin $\mathrm{E}$ receptor subtype EP4 in colon carcinogenesis. Cancer Res 62:28-32

5. Terada N, Shimizu Y, Kamba T, Inoue T, Maeno A, Kobayashi T, Nakamura E (2010) Identification of EP4 as a potential target for the treatment of castration-resistant prostate cancer using a novel xenograft model. Cancer Res 70:1606-1615
6. Kim J, Lakshmikanthan V, Frilot N, Daaka Y (2010) Prostaglandin E2 promotes lung cancer cell migration via EP4-B arrestin1-c-Src signalsome. Mol Cancer Res 8:569-577

7. Zhang Y, Ritzenthaler J, Sun X, Roman J, Han S (2009) Prostaglandin E2 stimulates human lung carcinoma cell growth through induction of integrin-linked kinase: the involvement of EP4 and Sp1. Cancer Res 69:896-904

8. Buchanan FG, Gorden DL, Matta P, Shi Q, Matrisian LM, DuBois RN (2006) Role of $\beta$-arrestin 1 in the metastatic progression of colorectal cancer. Proc Natl Acad Sci USA 103: 1492-1499

9. Chell SD, Witherden IR, Dobson RR, Moorghen M, Herman AA, Qualtrough D, Williams AC, Paraskeva C (2006) Increased EP4 receptor expression in colorectal cancer progression promotes cell growth and anchorage independence. Cancer Res 66:3106-3113

10. Rundhaug JE, Simper MS, Surh I, Fischer SM (2011) The role of the EP receptors for prostaglandin E2 in skin and skin cancer. Cancer Metastasis Rev 30:465-480

11. Wu J, Zhang Y, Frilot N, Kim JI, Kim W-J, Daaka Y (2011) Prostaglandin E2 regulates renal cell carcinoma invasion through the EP4 receptor-Rap GTPase signal transduction pathway. J Biol Chem 286:33954-33962

12. Subbaramaiah K, Hudis C, Chang S-H, Hla T, Dannenberg AJ (2008) EP2 and EP4 receptors regulate aromatase expression in human adipocytes and breast cancer cells: evidence of a BRCA1 and p300 exchange. J Biol Chem 283:3433-3444

13. Ma X, Kundu N, Rifat S, Walser T, Fulton AM (2006) Prostaglandin E receptor EP4 antagonism inhibits breast cancer metastasis. Cancer Res 66:2923-2927

14. Fulton AM, Ma X, Kundu N (2006) Targeting prostaglandin E EP receptors to inhibit metastasis. Cancer Res 66:9794-9797

15. Timoshenko A, Guoziong X, Chakrabarti S, Lala P, Chakraborty C (2003) Role of prostaglandin E2 receptors in migration of murine and human breast cancer cells. Exp Cell Res 289:265-274

16. Robertson FM, Simeone AM, Mazumdar A, Shah AH, McMurray JS, Ghosh S et al (2008) Molecular and pharmacological blockade of the EP4 receptor selectively inhibits both proliferation and invasion of human inflammatory breast cancer cells. J Exp Ther Oncol 7:299-312

17. Yang L, Huang Y, Porta R, Yanagisawa K, Gonzalez A, Segi E et al (2006) Host and direct antitumor effects and profound reduction in tumor metastasis with selective EP4 receptor antagonism. Cancer Res 66:9665-9672

18. Li JH, Reinhardt F, Herschman HR, Weinberg RA (2012) Cancer-stimulated mesenchymal stem cells create a carcinoma stem cell niche via prostaglandin $E_{2}$ signaling. Cancer Discov 2: $840-855$

19. Murase A, Taniguchi Y, Tonai-Kachi H, Nakao K, Takada J (2007) In vitro pharmacological characterization of CJ-042794, a novel, potent, and selective prostaglandin EP(4) receptor antagonist. Life Sci 82:226-232

20. Ma X, Kundu N, Collin PD, Goloubeva O, Fulton AM (2011) Frondoside A inhibits breast cancer metastasis and antagonizes prostaglandin E receptors EP4 and EP2. Breast Cancer Res Treat 132:1001-1008

21. Kundu N, Ma X, Walser T, Goloubeva O, Ostrand-Rosenberg S, Fulton AM (2009) Antagonism of the prostaglandin E receptor EP4 inhibits metastasis and enhances NK function. Breast Cancer Res Treat 117:235-242

22. Ma X, Kundu N, Ioffe O, Goloubeva O, Konger R, Baquet C, Gimotty P, Fulton AM (2010) Prostaglandin E receptor EP1 suppresses metastasis, is associated with better survival and may contribute to breast cancer disparities. Mol Cancer Res 8: $1310-1318$ 
23. Ma X, Holt D, Kundu N, Reader J, Goloubeva O, Take Y, Fulton AM (2013) A prostaglandin E (PGE) receptor EP4 antagonist protects natural killer cells from $\mathrm{PGE}_{2}$-mediated immunosuppression and inhibits breast cancer metastasis. Oncoimmunology 2:e22647

24. Al Hajj M, Wicha MS, Benito-Hernandez A, Morrison SJ, Clarke MF (2003) Prospective identification of tumorigenic breast cancer cells. Proc Natl Acad Sci USA 100:3983-3988

25. Weng D, Penzner JH, Song B, Koido S, Calderwood SK, Gong J (2012) Metastasis is an early event in mouse mammary carcinomas and is associated with cells bearing stem cell markers. Breast Cancer Res 14:R18

26. Conley SJ, Gheordunescu E, Kakarala P, Newman B, Korkaya H, Heath AN, Clouthier SG, Wicha MS (2012) Antiangiogenic agents increase breast cancer stem cells via the generation of tumor hypoxia. Proc Natl Acad Sci USA 109:2784-2789

27. Kenny PA, Lee GY, Myers CA, Neve RM, Semeiks JR et al (2007) The morphologies of breast cancer cell lines in threedimensional assays correlate with their profiles of gene expression. Mol Oncol 1:84-96

28. Fillmore CM, Kuperwasser C (2008) Human breast cancer cell lines contain stem-like cells that self-renew, give rise to phenotypically diverse progeny and survival chemotherapy. Breast Cancer Res 10:R25

29. Holt D, Ma X, Kundu N, Fulton AM (2011) Prostaglandin E2 (PGE2) suppresses natural killer cell function through the PGE2 receptor EP4. Cancer Immunol Immunother 60:1577-1586
30. Holt DH, Ma X, Kundu N, Collin PD, Fulton AM (2012) Modulation of host natural killer cell functions in breast cancer via prostaglandin E2 receptors EP2 and EP4. J Immunother 35:179-188

31. Boniface K, Bak-Jensen K, Li Y, Blumenschein W, McGeachy M, McClanahan T et al (2009) Prostaglandin E2 regulates Th17 cell differentiation and function through cyclic AMP and EP2/ EP4 receptor signaling. J Exp Med 206:535-548

32. Sharma S, Yang S, Zhu L, Reckamp K, Gardner B, Baratelli F (2005) Tumor cyclooxygenase-2/prostaglandin E2-dependent promotion of FOXP3 expression and CD4 $+\mathrm{CD} 25+\mathrm{T}$ regulatory cell activities in lung cancer. Cancer Res 65:5211-5225

33. Yao C, Sakata D, Esaki Y, Li Y, Matsuoka T, Kuroiwa K et al (2009) Prostaglandin E2-EP4 signaling promotes immune inflammation through Th1 cell differentiation and Th17 cell expansion. Nat Med 15:633-640

34. Rudnick JA, Arendt LM, Klebba I, Hinds JW, Iyer V, Gupta PB, Naber SP, Kuperwasser C (2011) Functional heterogeneity of breast fibroblasts is defined by a prostaglandin secretory phenotype that promotes expansion of cancer-stem like cells. PLoS One 6:e24605

35. Singh B, Cook DR, Vincent L, Hall CS, Lucci A (2011) Role of COX-2 in tumorospheres derived from a breast cancer cell line. J Surg Res 168:e39-e49 